\title{
Probing Atom-Surface Interactions by Diffraction of Bose-Einstein Condensates
}

\author{
Helmar Bender, Christian Stehle, Claus Zimmermann, and Sebastian Slama* \\ Physikalisches Institut and Center for Collective Quantum Phenomena in LISA+, Universität Tübingen, \\ Auf der Morgenstelle 14, D-72076 Tübingen, Germany \\ Johannes Fiedler, and Stefan Scheel \\ Institut für Physik, Universität Rostock, Universitätsplatz, 3, D-18055 Rostock, Germany \\ Stefan Yoshi Buhmann ${ }^{\dagger}$ \\ Quantum Optics and Laser Science, Blackett Laboratory, Imperial College London, Prince Consort Road, \\ London SW7 2AZ, United Kingdom \\ Valery N. Marachevsky ${ }^{\ddagger}$ \\ Department of Physics, Saint Petersburg State University, Ulianovskaya 1, \\ Petrodvorets, 198504 St. Petersburg, Russia
}

(Received 8 May 2013; revised manuscript received 7 October 2013; published 27 February 2014)

\begin{abstract}
In this article, we analyze the Casimir-Polder interaction of atoms with a solid grating and the repulsive interaction between the atoms and the grating in the presence of an external laser source. The CasimirPolder potential is evaluated exactly in terms of Rayleigh reflection coefficients and via an approximate Hamaker approach. The laser-tuned repulsive interaction is given in terms of Rayleigh transmission coefficients. The combined potential landscape above the solid grating is probed locally by diffraction of Bose-Einstein condensates. Measured diffraction efficiencies reveal information about the shape of the potential landscape in agreement with the theory based on Rayleigh decompositions.
\end{abstract}

DOI: 10.1103/PhysRevX.4.011029

The Casimir-Polder (CP) interaction is one of a class of examples where fluctuating electromagnetic fields give rise to (normally attractive) forces between matter $[1,2]$. For infinitely extended plane surfaces, $\mathrm{CP}$ forces can be readily calculated from the polarizability of the atom and the dielectric properties of the substrate [3] and have been measured in a number of experiments [4-11]. However, of particular importance is the influence of the surface geometry [12-14]. Nontrivial geometries can have a large impact on the exact force profile and can potentially be used for manipulating the closely related Casimir forces [15]. The possibility of tailoring the Casimir force is also of importance for applications in the microelectromechanical systems (MEMS) and nanoelectromechanical systems (NEMS) industry, where it is one of the limiting factors in the miniaturization of micromachines and microsensors [16].

\footnotetext{
*sebastian.slama@uni-tuebingen.de

†s.buhmann@imperial.ac.uk

*maraval@mail.ru
}

Published by the American Physical Society under the terms of the Creative Commons Attribution 3.0 License. Further distribution of this work must maintain attribution to the author(s) and the published article's title, journal citation, and DOI.
Subject Areas: Atomic and Molecular Physics, Nanophysics, Optics

One class of nontrivial geometries that have been investigated theoretically, both in the framework of atom-surface and surface-surface interactions, are periodic structures such as gratings [17-19]. Please note that the reflection of fast atoms from crystalline planar surfaces can also be understood as diffraction caused by the periodic shape of the attractive Casimir-Polder interaction. Corresponding experiments with hot atomic beams were reported some time ago [20]. In recent experiments, CP forces above gratings were measured by different methods [21-25]. In those experiments, the power-law coefficients describing the $\mathrm{CP}$ potential in the electrostatic and in the retarded regimes were determined. Recently, theoretical proposals have been put forward to measure the $\mathrm{CP}$ potential at corrugated surfaces with Bose-Einstein condensates [26].

In this article, we present both theoretical simulations and experimental measurements of the potential landscape for a single atom that is positioned at a submicron distance from a grating of metal nanowires. The potential landscape is composed of an attractive contribution due to the $\mathrm{CP}$ force and a repulsive contribution due to an evanescent light wave (EW) at the surface. The quantitative agreement between theory and experiment in the absence of free parameters demonstrates that complex surface-assisted 
potentials resulting from a combination of geometric structure and tunable evanescent laser potentials are now well understood both theoretically and experimentally. Whereas in previous experiments the measured values of the $\mathrm{CP}$ potential represent only an average over the complicated potential landscape above the structures, in this work we fully account for the dependence of the potential on the lateral position above such a surface.

The evanescent light wave is generated by the internal total reflection of a laser beam in the dielectric substrate carrying the grating. A repulsion from the surface is achieved by a laser $(\lambda=765 \mathrm{~nm})$ that is blue detuned with respect to the transition frequency of the atoms $\left(\mathrm{Rb}: \lambda_{0}=780 \mathrm{~nm}\right)$. Recently, we used this setup and enhanced the evanescent waves by exciting surface plasmon polaritons at the surface [27]. In a similar way, nanoobjects have been optically trapped in a patterned light field above a structured metallic film [28]. Here, we exploit the fact that the exact shape of the total potential landscape can be tuned by the strength of the optical dipole potential via the laser intensity. This allows us to acquire spatially resolved information of the surface potentials: Fig. 1 shows a simulation of the potential landscape for a typical laser power of $P=211 \mathrm{~mW}$, including the optical dipole potential of the evanescent wave and the Casimir-Polder potential.

The CP potential with its strong attraction towards the gold stripes and the repulsive EW potential with its maximal repulsion above the sapphire surface combine to a periodic potential landscape that resembles a chain of

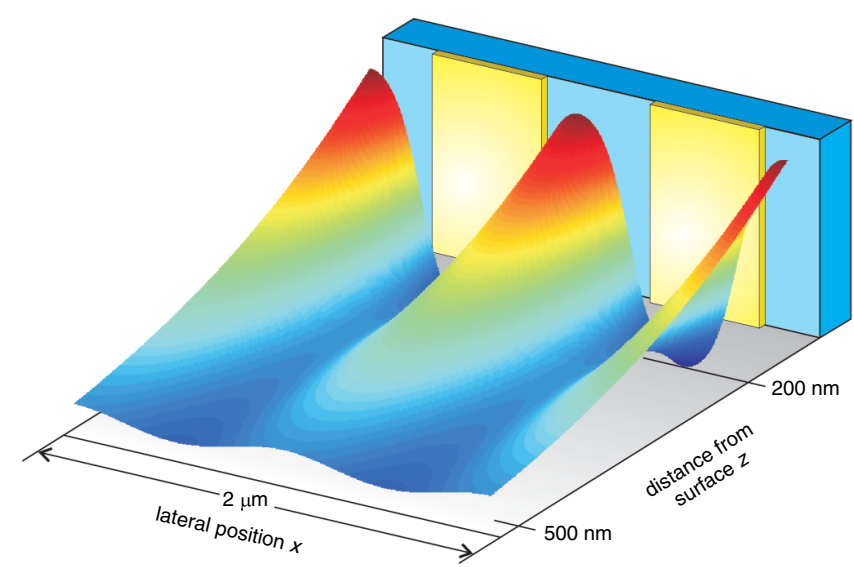

FIG. 1 Simulation of the combined potential landscape, including the evanescent-wave potential and Casimir-Polder landscape as calculated from Eqs. (1) and (2) for a laser power of $P=211 \mathrm{~mW}$. The repulsive evanescent-wave potential is generated by a laser beam that is reflected by the total internal reflection in the substrate. The gold stripes weaken the intensity of the evanescent wave and thus modulate the strength of the repulsion. The attractive Casimir-Polder potential reaches its maximum value above the gold stripes and further increases this modulation. At a distance of $z=200 \mathrm{~nm}$, the potential is laterally modulated with an amplitude of $\Delta E / k_{\mathrm{B}}=14 \mu \mathrm{K}$. hills in front of the grating surface with valleys that lead to the centers of the gold stripes. The heights and widths of the hills depend on the laser power (Fig. 2), with larger powers resulting in higher and broader hills.

The EW potential

$$
U_{\mathrm{EW}}(\boldsymbol{r})=\sum_{i=1,2} \frac{\left|\boldsymbol{d}_{i}\right|^{2}|\boldsymbol{E}(\boldsymbol{r})|^{2}}{3 \hbar \Delta_{i}}
$$

is the potential due to the external monochromatic electric field $\boldsymbol{E}$ with its frequency $\omega$ close to a specific set of atomic transitions of $\mathrm{Rb}$ with dipole matrix elements $\boldsymbol{d}_{i}$ and detunings $\Delta_{i}=\omega-\omega_{i}$. It is dominated by these atomic transitions and the transmission properties of the grating at a single laser frequency $\omega$. The EW potential is expressed in terms of Rayleigh transmission coefficients in the Appendix.

In the simulations, the ground-state $\mathrm{CP}$ potential of the $\mathrm{Rb}$ atoms is calculated to lowest order in their isotropic ground-state polarizability as [29]

$$
U_{\mathrm{CP}}(\boldsymbol{r})=\frac{\hbar \mu_{0}}{2 \pi} \int_{0}^{\infty} d \xi \xi^{2} \alpha(\mathrm{i} \xi) \operatorname{Tr} \mathbf{G}^{(1)}(\boldsymbol{r}, \boldsymbol{r}, \mathrm{i} \xi) .
$$

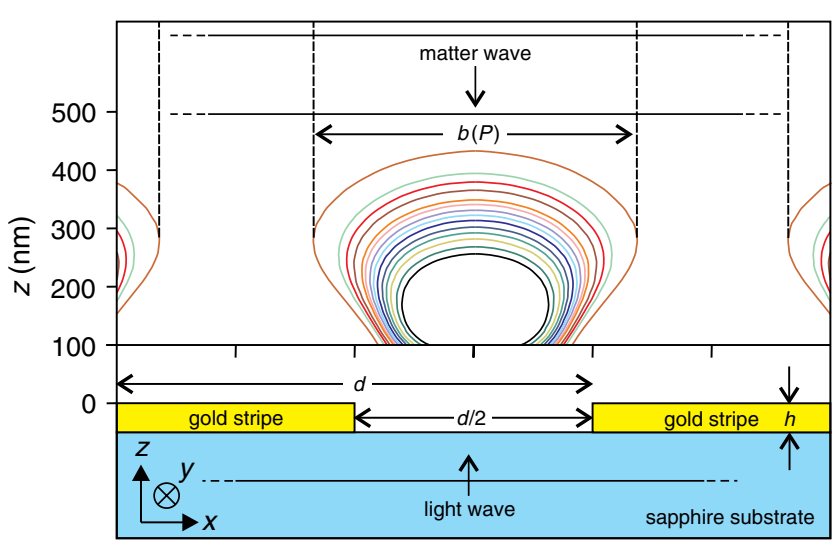

FIG. 2 Geometry of the metal grating. Approximately 200 gold stripes with $h=50 \mathrm{~nm}$ height and $500 \mathrm{~nm}$ width are deposited on a sapphire substrate and form a grating with a $d=1 \mu \mathrm{m}$ period. A laser beam impinges under an angle of $\theta=35.50^{\circ}$ from the sapphire substrate onto the interface to vacuum (respectively, gold) with its $k$ vector in the $(y, z)$ plane. The laser beam is internally reflected and generates an evanescent wave. The combination of the repulsive potential due to the evanescent wave with the Casimir-Polder interaction forms a potential landscape above the grating. The colored lines (black to brown, corresponding to laser powers $P=120,126,133,138$, $144,151,156,162,169,174,187,198,211,247 \mathrm{~mW})$ are simulations of equipotential lines using exact theory based on Rayleigh decompositions for a ${ }^{87} \mathrm{Rb}$ atom moving towards the surface with velocity $v=3.4 \mathrm{~cm} / \mathrm{s}$. From those, we deduce the width $b(P)$ where atoms are reflected. The two horizontal lines at the top represent the incoming matter wave and are drawn at a distance given by the de Broglie wavelength $\lambda_{\mathrm{dB}}=\frac{2 \pi \hbar}{m v}=$ $135 \mathrm{~nm}$, with atomic mass $m$. 
Here, the polarizability $\alpha(\mathrm{i} \xi)$ is approximated by an eightline model (see the Appendix), and $\mathbf{G}^{(1)}$ is the scattering Green tensor which, for the grating structure in Fig. 2, can be given as a Rayleigh decomposition in terms of Rayleigh reflection coefficients. Because of the integral over all imaginary frequencies as a result of the vacuum fluctuations of the electromagnetic field, the $\mathrm{CP}$ potential depends on all atomic transition frequencies and all eigenfrequencies of the macroscopic system (grating).

From the theoretical point of view, it is quite interesting to perform a comparison of an exact approach to the calculation of CP potentials near gratings with an approximate Hamaker approach [30,31], which is based on a pairwise summation of van der Waals forces between volume elements of one body with those of the other. However, such an approach neglects many-body interactions that can lead to wrong results, in particular, for complex geometric structures $[32,33]$. The nonadditivity of Casimir forces induced by many-body interactions [34] implies that the position, shape, and material dependencies of such forces are intertwined in a complicated way. Nevertheless, the Hamaker approach is widely used in applications such as colloid science and biology [35]. The most prominent example is the adhesive force of gecko feet [36].

Surprisingly, so far not a single experiment has addressed the accuracy of the Hamaker approach in atom-surface interactions, despite there being a large amount of work that has addressed deviations of the proximity force approximation (PFA) from the exact calculations, taking diffraction into account in the context of surface-surface interactions [33,37-41]. In our case, the $\mathrm{CP}$ potential contribution to the combined potential $(\mathrm{CP}+\mathrm{EW})$ is relevant at given separations, and one can discern the difference between the result of exact simulation of the Casimir-Polder potential expressed in terms of Rayleigh coefficients and the result of an approximate Hamaker approach.

The $\mathrm{CP}$ potential in the Hamaker approach is calculated in the local-field-corrected first-order Born approximation as

$$
\begin{aligned}
\mathbf{G}^{(1)}(\boldsymbol{r}, \boldsymbol{r}, \omega)= & \frac{\omega^{2}}{c^{2}} \frac{\chi(\omega)}{1+\chi(\omega) / 3} \\
& \times \int \mathrm{d}^{3} s \mathbf{R}^{(0)}(\boldsymbol{r}, \boldsymbol{s}, \omega) \mathbf{R}^{(0)}(\boldsymbol{s}, \boldsymbol{r}, \omega),
\end{aligned}
$$

where $\mathbf{R}^{(0)}(\boldsymbol{r}, \boldsymbol{s}, \omega)$ is the regular part of the Green tensor, $\chi(\omega)$ is the susceptibility of the gold stripes, and the integration extends over the total volume $V$ of the grating. Details of all the calculations are contained in the Appendix.

Experimentally, we probe the width $b$ of the reflection zones in Fig. 2 by reflecting Bose-Einstein condensates (BEC) from the surface. The experiment is carried out as follows. A BEC is prepared in a magnetic trap at a distance of several hundred $\mu \mathrm{m}$ from the grating and accelerated such that it moves towards the surface with a constant velocity $v=3.4 \mathrm{~cm} / \mathrm{s}$. Thereby, the cloud is ballistically expanding and transforms its interaction energy in velocity spread. For that reason, interaction effects do not play a role during reflection from the surface. The experimental details of this preparation are contained in the Appendix. The atoms reflect from the surface only at those lateral positions where the potential height exceeds the kinetic energy of the atoms. This happens in a zone with width $b$ in each lattice site (see Fig. 2). Note that considerable quantum reflection of $\mathrm{Rb}$ atoms at the $\mathrm{CP}$ potential of a solid surface would require atomic velocities below a few $\mathrm{mm} / \mathrm{s}$ [9]. For the velocity of $v=3.4 \mathrm{~cm} / \mathrm{s}$, it is completely negligible. By tuning the laser power, the reflection zone width $b$ is changed and different distances from the surface are probed. Each atom of the BEC approaching the surface constitutes a matter wave with a lateral extension that is given by the size of the BEC on the order of several tens of microns. This size is much larger than the grating period; thus, the matter wave is diffracted from the periodic structure of reflection zones in a direction $x$ of period $d$.

In a simplified model that neglects the curvature of the equipotential lines, we consider reflection of the matter wave from the same reflection zones of width $b$ as for a single atom. The resulting atomic momentum distribution in the far field is analogous to Fraunhofer diffraction. For an arrangement of rectangular stripes as shown in Fig. 2, the occupation $p_{n}$ of diffraction order $n$ (normalized to $p_{0}$ ) is given by

$$
p_{n}=\left|\operatorname{sinc}\left(\pi n \cdot \frac{b}{d}\right)\right|^{2}
$$

It depends only on the ratio $b / d$. This is illustrated in the theoretical curves in Fig. 3, where we plot the relative occupations (normalized to $\sum p_{n}$ ) of the diffraction orders as determined from Eq. (4). In the limit of $b / d \rightarrow 0$, the situation resembles the emission of waves from a chain of pointlike sources, in which all diffraction orders are equally occupied. In contrast, the limit $b / d \rightarrow 1$ corresponds to a reflection from a surface with a constant density profile. Here, the atomic cloud remains fully in the diffraction order $n=0$.

In the experiment, we analyze the relative occupation of individual diffraction orders by measuring the momentum distribution $p_{x}=\hbar k_{x}$ of the atoms. This is done by taking an absorption image of the cloud after ballistic expansion for a time of flight of $t_{\text {tof }}=21.5 \mathrm{~ms}$ after reflection from the surface. A typical image is shown in the inset of Fig. 3. From the image, the atom numbers $N_{n}$ corresponding to diffraction orders $n$ with momentum $p_{x, n}=n \hbar \frac{2 \pi}{d}$ are counted within the yellow boxes and are scaled to the total number of reflected atoms. This provides us data 


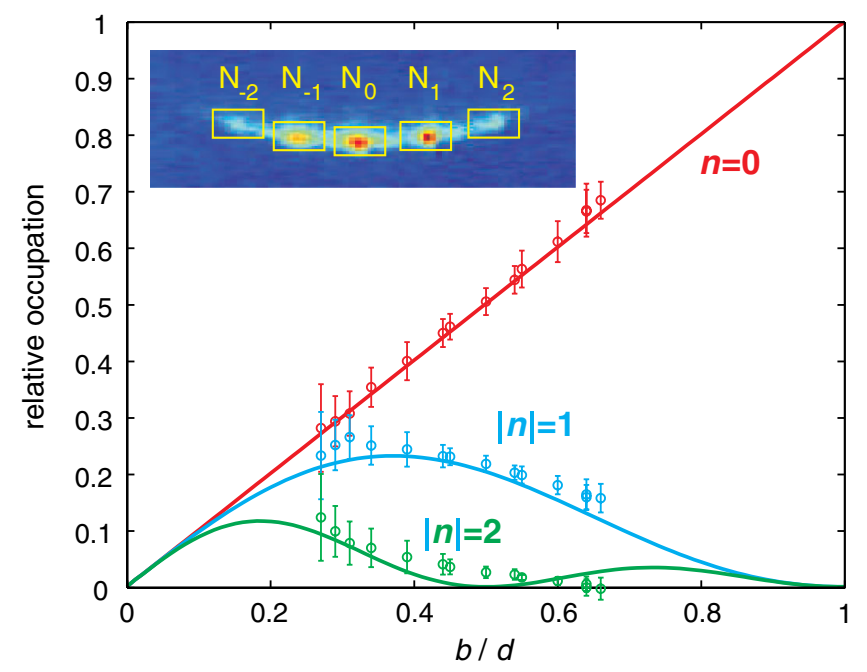

FIG. 3 Matter-wave diffraction. Relative occupation of diffraction orders as a function of the relative width of the reflection zone $b / d$. The curves are obtained from Eq. (4). Data points represent the measured occupation of diffraction orders as shown in the inset. The horizontal position of each triple of data points (its value $b / d$ ) is obtained from a fit to the theoretical curves.

triples of relative populations of diffraction orders $n=0$, $\pm 1, \pm 2$ for each value of laser power. The populations for $n \neq 0$ are averaged over the populations of the orders with $\pm n$. Each triple is individually fitted by the theoretical curves in Fig. 3 and is thus attributed a certain value of $b / d$.

Although all data points coincide with the corresponding theory curves very well, it is notable that the model used is valid only for the lower laser powers used, corresponding to the small values of $b / d$. The model is based on amplitude modulation only and thus neglects phase modulation of the reflected matter wave. As shown in the Appendix, this approximation is valid for laser powers $P \lesssim 140 \mathrm{~mW}$, whereas phase imprinting becomes an important effect for the large laser powers used. Because a complete calculation of the diffraction problem is more difficult than electromagnetic diffraction and would not affect the overall meaning of the results presented here, we restrict the conclusions of this work to the data points with small laser power.

The fitted values of $b / d$ are now compared with the theoretical prediction that is accessible from the width of the equipotential lines shown in Fig. 2. As can be seen in Fig. 4, the measured diffraction cannot be explained by the spatial modulation of the optical potential alone. Thus, the experiment is sensitive to the Casimir-Polder potential. Moreover, it can partly discriminate between different theoretical models: The data points agree with exact theory based on Rayleigh decompositions within their statistic and systematic errors. The Hamaker approach underestimates the strength of the Casimir-Polder potential. The corresponding values of $b / d$ for low values of $P$ in Fig. 4 are

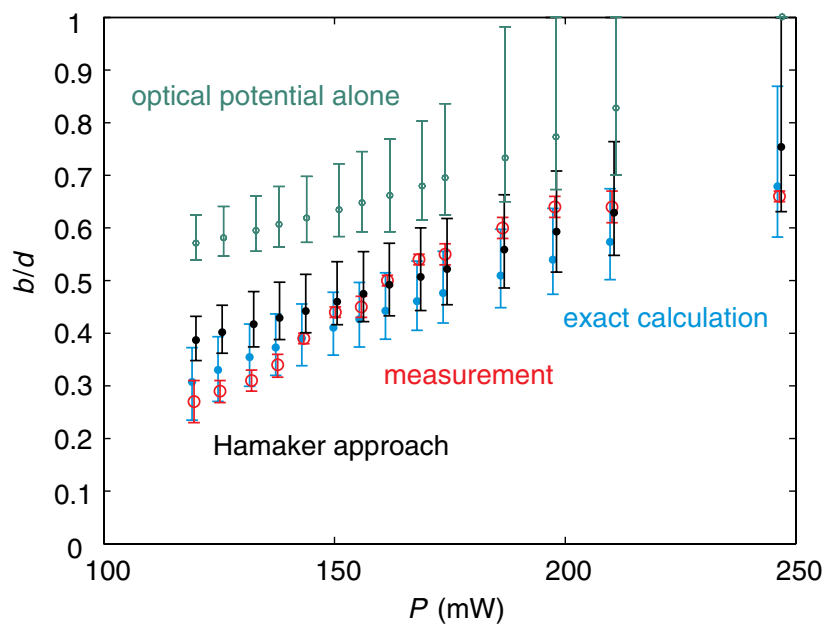

FIG. 4 Comparison between theory and experiment. Width of the reflection zone $b / d$ versus laser power $P$. Red circles are experimental data points obtained from the fit in Fig. 3. Error bars of the data points are due to the combined statistic and systematic uncertainties in the atom-number measurement. Blue dots show the theoretical values taken from the equipotential lines in Fig. 2 and represent the result of the exact calculation based on Rayleigh decompositions. Black points are the corresponding results based on a Hamaker approach. Green points stem from a simulation of the optical potential alone, neglecting any influence of the Casimir-Polder potential. Error bars of the theoretical points represent the systematic error due to the uncertainty in the laser intensity at the surface $\Delta I= \pm 5 \%$ and the velocity of the atoms $\Delta v= \pm 0.3 \mathrm{~cm} / \mathrm{s}$. Please note that the data points are horizontally shifted one line width $(\lesssim 1 \mathrm{~mW})$ for clarity.

thus larger than the observed data points and deviate from them by more than 1 standard deviation. In the range of large $P$ in Fig. 4, the optical potential dominates over the $\mathrm{CP}$ potential and reduces the difference between exact theory and the Hamaker model.

It is obvious from Fig. 4 that the functional profile of the data points differs from that of the simulations for laser powers $P \gtrsim 140 \mathrm{~mW}$. In particular, the value of $b / d$ of the measured data points saturates for large laser powers. This observation is caused by the growing influence of phase imprinting for increasing laser power, as shown in the Appendix. This leads to a principally different behavior of the scattered field, which manifests e.g., in the absence of missing orders [42]. Qualitatively, for high reflectivity, the assumption of instantaneous reflection is not justified. Instead, the interaction time of the atoms with the surface potential and the strength of the latter depend on the lateral position $x$; i.e., depending on the lateral position, the matter wave acquires a different phase. A periodic potential imprints a phase that leads to a substantial diffraction even when all atoms are reflected and thus simulates a saturation of $b / d$ for large laser powers [43-45].

It is worth considering the possible influence of surface potential generated by $\mathrm{Rb}$ atoms adsorbed to our grating. As reported in Ref. [46], surface potentials are primarily 
generated on metal surfaces such as the grating bars. The authors report an electric field of $1 \mu \mathrm{V} / \mathrm{cm}$ per adsorbed atom at a distance $z=10 \mu \mathrm{m}$, with a $1 / z^{2.3}$ distance dependence, corresponding to roughly $0.1 \mathrm{~V} / \mathrm{m}$ at a distance of $430 \mathrm{~nm}$. At this distance, adsorbed atoms from a surface area of about $1 \mu \mathrm{m} \times 1 \mu \mathrm{m}=1 \mu \mathrm{m}^{2}$ contribute to the field. The $2 \times 10^{5}$ atoms involved in a single run of the experiment are spread out over a cloud of dimensions $100 \mu \mathrm{m} \times 50 \mu \mathrm{m}=5000 \mu \mathrm{m}^{2}$ so that $2 \times 10^{5} \times(1 / 5000) \times$ $(1 / 2)=20$ atoms potentially impinge on this area, where the factor $1 / 2$ represents the filling factor of the grating. With the small sticking probability of $0.8 \%$ as observed in Ref. [47], 1600 atoms could accumulate in the relevant surface area in the course of 10000 runs of the experiment. This would lead to a total electric field of the order of $2 \times 10^{2} \mathrm{~V} / \mathrm{m}$, much smaller than the value of the evanescent laser field of about $3 \times 10^{4} \mathrm{~V} / \mathrm{m}$ at the same distance calculated for a laser power of $247 \mathrm{~mW}$. Alternative measurements [48] predict even smaller values of the electric fields due to adsorbed atoms.

In conclusion, we have experimentally probed surface potential landscapes that are composed of Casimir-Polder forces and optical dipole forces above metallic nanostructures. We have used matter-wave diffraction of Bose-Einstein condensates as a measuring tool, which, in principle, can be applied to arbitrary surfaces. Complementary to previous experiments in which spatial averages of the Casimir-Polder coefficients were determined, we obtain additional spatial information by analyzing the occupation of individual diffraction orders. Our data agree quantitatively with numerical calculations of the surface potentials (1) and (2) based on exact theory where potentials are expressed in terms of Rayleigh coefficients, whereas a Hamaker approach leads to incompatible results for low laser powers. The difference is so large that an experiment can distinguish between both approaches. From the theoretical point of view, the main result is the first comparison of exact theoretical results for atom-surface potentials above a structured surface (based on Rayleigh decompositions) and experimental data used to measure the structure of the potential landscape. The results open a wide range of possibilities for exact theoretical simulations and design of atomic-surface potentials in the vicinity of nanostructured and microstructured surfaces.

The fact that we understand these potentials very well is crucial for the design and realization of nanoscale surface traps for surface quantum optics experiments with cold atoms. Our analysis demonstrates the possibility to couple atoms to light near structured surfaces in a controlled way. Moreover, the metallic parts of the surface can give rise to spectrally broad surface plasmon resonances in the opticalfrequency range. Related phonon polariton resonances in the infrared frequency range have e.g., led to the observation of repulsive Casimir-Polder forces of highly excited Cs atoms [49]. A plasmon-based repulsive Casimir-Polder force would give a perspective on fascinating scenarios for controlling $\mathrm{CP}$ forces [50] and for generating surface traps for cold atoms that do not require external magnetic or optical fields.

C. S. was supported by Carl-Zeiss Stiftung BadenWürttemberg. Support from the European Science Foundation (ESF) within the activity "New Trends and Applications of the Casimir Effect" is gratefully acknowledged. This work was financially supported by the UK EPSRC. The research leading to these results has received funding from the European Union Seventh Framework Programme (FP7-PEOPLE-2010-IRSES) under Grant Agreement No. PIRSES-GA-2010-268717. V. N. M was partially supported by the Saint Petersburg State University Grant No. 11.38.660.2013. Furthermore, we acknowledge support by the Deutsche Forschungsgemeinschaft within the European Collaborative Research program of the European Science Foundation.

\section{APPENDIX}

\section{Simulation of the Casimir-Polder potential}

The ground-state CP potential of an atom or molecule can be given as [29]

$$
U_{\mathrm{CP}}(\boldsymbol{r})=\frac{\hbar \mu_{0}}{2 \pi} \int_{0}^{\infty} d \xi \xi^{2} \operatorname{Tr}\left[\boldsymbol{\alpha}(\mathrm{i} \xi) \cdot \mathbf{G}^{(1)}(\boldsymbol{r}, \boldsymbol{r}, \mathrm{i} \xi)\right] .
$$

To allow for potentially anisotropic molecules, we have included the ground-state polarizability tensor $\boldsymbol{\alpha}(\mathrm{i} \xi) ; \mathbf{G}^{(1)}$ is the scattering Green tensor. We are neglecting thermal contributions to the $\mathrm{CP}$ force, which is a good approximation for the distances considered in this work. For the structure in Fig. 2, the scattering Green tensor can be given in a Rayleigh decomposition as

$$
\begin{aligned}
\mathbf{G}^{(1)}\left(\boldsymbol{r}, \boldsymbol{r}^{\prime}, \omega\right)= & \frac{\mathrm{i}}{8 \pi^{2}} \int_{-\pi / d}^{\pi / d} \mathrm{~d} k_{x} \sum_{m, n=-\infty}^{\infty} \int_{-\infty}^{\infty} \mathrm{d} k_{y} \\
& \times \sum_{\sigma, \sigma^{\prime}=E, H} \frac{\mathrm{e}^{\mathrm{i}\left(k_{x}^{m} x-k_{x}^{n} x^{\prime}\right)+\mathrm{i} k_{y}\left(y-y^{\prime}\right)+\mathrm{i}\left(k_{z}^{m} z+k_{z}^{n} z^{\prime}\right)}}{k_{z}^{n}} \\
& \times \boldsymbol{e}_{m+}^{\sigma}\left(k_{x}, k_{y}, \omega\right) R_{m n}^{\sigma \sigma^{\prime}}\left(k_{x}, k_{y}, \omega\right) \boldsymbol{e}_{n-}^{\sigma^{\prime}}\left(k_{x}, k_{y}, \omega\right) .
\end{aligned}
$$

Here, $\boldsymbol{k}=\left(k_{x}^{m}, k_{y}, k_{z}^{m}\right)$ is the wave vector; its $x$ and $z$ components read $k_{x}^{m}=k_{x}+m q(q=2 \pi / d$ : lattice vector of the grating) and $k_{z}^{m}=\sqrt{\omega^{2} / c^{2}-\left(k_{x}^{m}\right)^{2}-k_{y}^{2}}$, with Im $k_{z}^{m}>0$. The polarization unit vectors for upward or downward moving waves are defined by 


$$
\begin{gathered}
\boldsymbol{e}_{m \pm}^{E}\left(k_{x}, k_{y}, \omega\right)=\frac{c}{\omega \sqrt{\omega^{2} / c^{2}-k_{y}^{2}}}\left(\begin{array}{c}
k_{x}^{m} k_{y} \\
k_{y}^{2}-\omega^{2} / c^{2} \\
\pm k_{y} k_{z}^{m}
\end{array}\right), \\
\boldsymbol{e}_{m \pm}^{H}\left(k_{x}, k_{y}, \omega\right)=\frac{1}{\sqrt{\omega^{2} / c^{2}-k_{y}^{2}}}\left(\begin{array}{c}
\mp k_{z}^{m} \\
0 \\
k_{x}^{m}
\end{array}\right) .
\end{gathered}
$$

The latter are chosen such that the $y$ components of the electric or magnetic fields vanish for $\sigma=H, E$. The Rayleigh reflection coefficients $R_{m n}^{\sigma \sigma^{\prime}}\left(k_{x}, k_{y}, \omega\right)$ are calculated by numerically integrating the Maxwell equations within the grating $(0<z<h)$ and imposing conditions of continuity at its upper (free-space) and lower (sapphire) boundaries [17]. They are even functions of $k_{y}$ and obey the following symmetries: $R_{m n}^{\sigma \sigma^{\prime}}\left(k_{x}, k_{y}, \mathrm{i} \xi\right)= \pm R_{-m-n}^{\sigma \sigma^{\prime *}}\left(-k_{x}\right.$, $\left.k_{y}, \mathrm{i} \xi\right)$ and $R_{m n}^{\sigma \sigma^{\prime}}\left(k_{x}, k_{y}, \mathrm{i} \xi\right) \beta_{z}^{n}= \pm R_{-n-m}^{\sigma^{\prime} \sigma *}\left(k_{x}, k_{y}, \mathrm{i} \xi\right) \beta_{z}^{m}$, with $\beta_{z}^{n}=\sqrt{\xi^{2} / c^{2}+\left(k_{x}^{n}\right)^{2}+k_{y}^{2}}$, the sign + for $\sigma \sigma^{\prime}=E E, H H$, and the sign - otherwise. Our theory is able to allow for anisotropic atoms or molecules. For a sufficiently anisotropic molecule, we find a repulsive $\mathrm{CP}$ potential in particular spatial locations, similar to the repulsive Casimir force predicted in Ref. [15] (see also Refs. [51-53]). Note that for the isotropic atoms used in the current experiment, $\boldsymbol{\alpha}=\alpha \mathbf{l}$ (I: unit tensor), Eq. (A1) simplifies to Eq. (1) and our formalism reduces to that of Ref. [18].

\section{Simulation of the evanescent-wave potential}

The evanescent-wave potential is generated by an incoming wave of (free-space) wavelength $\lambda=765 \mathrm{~nm}$, which impinges on the sapphire-grating interface at an incidence angle $\theta=35.50^{\circ}=0.6196 \mathrm{rad}$, with the plane of incidence being parallel to the grating bars. The components of the wave vector in the sapphire layer are hence $k_{x}^{m}=0, \quad k_{y}=k \sin \theta=8.39 \times 10^{6} \mathrm{~m}^{-1}, \quad k_{z}^{0+}=$ $k \cos \theta=1.18 \times 10^{7} \mathrm{~m}^{-1}\left(k=\operatorname{Re} n_{\text {sapp }} \omega / c\right.$, with $\operatorname{Re} n_{\text {sapp }}=$ 1.76 and $\left.\omega=2 \pi c / \lambda=2.46 \times 10^{15} \mathrm{rad} / \mathrm{s}\right)$. The incoming field is polarized such that its component perpendicular to the grating vanishes; it can hence be written as $\boldsymbol{E}_{\text {in }}(\boldsymbol{r})=E_{\text {sapp }} \boldsymbol{e}_{0+}^{E}\left(0, k_{y}, \omega\right) \mathrm{e}^{\mathrm{i}\left(k_{y} y+k_{z}^{0+} z\right)}$. The field amplitude inside sapphire can be related to the respective laser power $P_{\text {sapp }}$ and beam waist $w_{\text {sapp }}$ via $\frac{1}{2} \epsilon_{0} n_{\text {sapp }} c E_{\text {sapp }}^{2}=I_{\text {sapp }}=$ $P_{\text {sapp }} /\left(2 \pi w_{\text {sapp }}^{2}\right)$. The laser power is in turn related to its free-space value by $P_{\text {sapp }}=T_{\text {free-space } \rightarrow \text { sapp }} P_{\text {free-space, }}$ where $T_{\text {free-space } \rightarrow \text { sapp }}=0.88$ has been determined experimentally from a setup with symmetric light paths. The measured beam waist of $170 \mu \mathrm{m}$ in free space results in an effective beam waist $w_{\text {sapp }}=182.9 \mu \mathrm{m}$ in sapphire after transitions through the free-space-glass and glass-sapphire interfaces. After transmission through the grating, the external laser leads to a field

$$
\begin{aligned}
\boldsymbol{E}(\boldsymbol{r})= & E_{\text {sapp }} \sum_{n=-\infty}^{\infty} \sum_{\sigma=E, H} \mathrm{e}^{\mathrm{i}\left(n q x+k_{y} y\right)-\kappa_{z}^{n+} z} \\
& \times \boldsymbol{e}_{n+}^{\sigma}\left(n q, k_{y}, \omega\right) T_{n 0}^{\sigma E}\left(0, k_{y}, \omega\right)
\end{aligned}
$$

with $\kappa_{z}^{n+}=\sqrt{k_{y}^{2}-\omega^{2} / c^{2}+n^{2} q^{2}}$, where the Rayleigh transmission coefficients $T_{m n}^{\sigma \sigma^{\prime}}\left(k_{x}, k_{y}, \omega\right)$ are found from numerical integration in the same way as the respective reflection coefficients. When interacting with a $\mathrm{Rb}$ atom, this evanescent field generates an optical potential

$$
\begin{aligned}
U_{\mathrm{EW}}(\boldsymbol{r})= & \sum_{i=1,2} \frac{\left|\boldsymbol{d}_{i}\right|^{2}|\boldsymbol{E}(\boldsymbol{r})|^{2}}{3 \hbar \Delta_{i}} \\
= & \sum_{i=1,2} \frac{\left|\boldsymbol{d}_{i}\right|^{2} E_{\mathrm{sapp}}^{2}}{3 \hbar \Delta_{i}} \\
& \times \sum_{m, n=-\infty}^{\infty} \sum_{\sigma, \sigma^{\prime}=E, H} \mathrm{e}^{\mathrm{i}(m-n) q x-\left(\kappa_{z}^{m+}+\kappa_{z}^{n+*}\right) z} \\
& \times \boldsymbol{e}_{m+}^{\sigma}\left(m q, k_{y}, \omega\right) \cdot \boldsymbol{e}_{n+}^{\sigma^{\prime *}}\left(n q, k_{y}, \omega\right) \\
& \times T_{m 0}^{\sigma E}\left(0, k_{y}, \omega\right) T_{n 0}^{\sigma^{\prime} E^{*}}\left(0, k_{y}, \omega\right) .
\end{aligned}
$$

\section{Details of calculations}

The potential $U_{\mathrm{EW}}(\boldsymbol{r})$ (1) was evaluated using transition frequencies $\omega_{1}=2.37 \times 10^{15} \mathrm{rad} / \mathrm{s}, \omega_{2}=2.41 \times 10^{15} \mathrm{rad} / \mathrm{s}$ and dipole-matrix elements $d_{1}=2.53 \times 10^{-29} \mathrm{Cm}, d_{2}=$ $3.57 \times 10^{-29} \mathrm{Cm}$ for the $D_{1}\left(5^{2} S_{1 / 2} \rightarrow 5^{2} P_{1 / 2}\right)$ and $D_{2}$ $\left(5^{2} S_{1 / 2} \rightarrow 5^{2} P_{3 / 2}\right)$ lines of the isotropic $\mathrm{Rb}$ atom [54,55]; one can neglect the contribution of other transition lines in Eq. (1).

In the calculation of the $\mathrm{CP}$ potential (2), the polarizability

$$
\alpha(i \omega)=\sum_{i=1}^{8} \frac{2 \omega_{i}\left|\boldsymbol{d}_{i}\right|^{2}}{3 \hbar\left(\omega_{i}^{2}+\omega^{2}\right)}
$$

for the isotropic $\mathrm{Rb}$ atom was evaluated within a model, taking into account eight transition frequencies [54]: $\omega_{1}=2.37 \times 10^{15} \mathrm{rad} / \mathrm{s}, \quad d_{1}=2.53 \times 10^{-29} \mathrm{Cm}, \quad \omega_{2}=$ $2.41 \times 10^{15} \mathrm{rad} / \mathrm{s}, \quad d_{2}=3.57 \times 10^{-29} \mathrm{Cm}, \quad \omega_{3}=4.468 \times$ $10^{15} \mathrm{rad} / \mathrm{s}, d_{3}=0.200 \times 10^{-29} \mathrm{Cm}, \omega_{4}=4.482 \times 10^{15} \mathrm{rad} / \mathrm{s}$, $d_{4}=0.324 \times 10^{-29} \mathrm{Cm}, \quad \omega_{5}=5.245 \times 10^{15} \mathrm{rad} / \mathrm{s}, \quad d_{5}=$ $0.069 \times 10^{-29} \mathrm{Cm}, \quad \omega_{6}=5.251 \times 10^{15} \mathrm{rad} / \mathrm{s}, \quad d_{6}=0.121 \times$ $10^{-29} \mathrm{Cm}, \omega_{7}=5.622 \times 10^{15} \mathrm{rad} / \mathrm{s}, d_{7}=0.035 \times 10^{-29} \mathrm{Cm}$, $\omega_{8}=5.625 \times 10^{15} \mathrm{rad} / \mathrm{s}, d_{8}=0.067 \times 10^{-29} \mathrm{Cm}$.

Distances in the range $\lambda_{8} \sim 300 \mathrm{~nm} \lesssim z \lesssim \lambda_{1} \sim 800 \mathrm{~nm}$ are in between the Casimir-Polder regime $\left(z \gg \lambda_{i}\right)$ and the nonretarded van der Waals regime $\left(z \ll \lambda_{i}\right)$ of the $\mathrm{CP}$ potential. In the range of separations $100 \mathrm{~nm} \leq z \lesssim \lambda_{i}$ used in the calculations, the frequencies $\omega \lesssim \omega_{i}$ yield the leading contribution to the integral (2). 
The numerical error in the evaluation of the combined potential (which is the sum of the Casimir-Polder and evanescent-wave potentials) is $0.1 \%$. The number of Rayleigh coefficients used was selected to obtain the needed accuracy; $N=30 \quad(2 N+1$ terms in every Rayleigh expansion) is sufficient to obtain the potential with the required accuracy even at closest separations from the grating, as shown in Fig. 2.

\section{Casimir-Polder potential using the Hamaker approach}

The scattering Green tensor $\mathbf{G}^{(1)}(\boldsymbol{r}, \boldsymbol{r}, \omega)$ in Eq. (A1) can be expanded in a Born series with respect to a known reference Green tensor $\mathbf{G}^{(0)}(\boldsymbol{r}, \boldsymbol{r}, \omega)$ as $[3,56]$

$$
\begin{aligned}
\mathbf{G}^{(1)}(\boldsymbol{r}, \boldsymbol{r}, \omega)= & \frac{\omega^{2}}{c^{2}} \int \mathrm{d}^{3} s \mathbf{G}^{(0)}(\boldsymbol{r}, \boldsymbol{s}, \omega) \delta \epsilon(\omega) \mathbf{G}^{(0)}(\boldsymbol{s}, \boldsymbol{r}, \omega) \\
& +\left(\frac{\omega^{2}}{c^{2}}\right)^{2} \int \mathrm{d}^{3} s \mathrm{~d}^{3} s^{\prime} \mathbf{G}^{(0)}(\boldsymbol{r}, \boldsymbol{s}, \omega) \delta \epsilon(\omega) \\
& \times \mathbf{G}^{(0)}\left(\boldsymbol{s}, \boldsymbol{s}^{\prime}, \omega\right) \times \delta \epsilon(\omega) \mathbf{G}^{(0)}\left(\boldsymbol{s}^{\prime}, \boldsymbol{r}, \omega\right)+\cdots,
\end{aligned}
$$

where the integration range covers the volume of the material under investigation. The perturbation $\delta \epsilon(\omega)=$ $\epsilon^{(1)}(\omega)-\epsilon^{(0)}(\omega)$ denotes the deviation from the reference permittivity. In the case of the free-space Green tensor as our reference, the difference permittivity simply equals the susceptibility [here, $\delta \epsilon(\omega)=\chi_{\mathrm{Au}}(\omega)$ ] of the material. The free-space Green tensor can be written as $(\boldsymbol{\varrho}=\boldsymbol{r}-\boldsymbol{s}$, $\varrho=|\varrho|)[3]$

$$
\begin{aligned}
\mathbf{G}^{(0)}(\boldsymbol{\varrho}, \omega)= & -\frac{c^{2}}{3 \omega^{2}} \boldsymbol{\delta}(\boldsymbol{\varrho}) \\
& +\frac{\omega}{4 \pi c}\left[f\left(\frac{c}{\omega \varrho}\right) \mathbf{I}-g\left(\frac{c}{\omega \varrho}\right) \frac{\boldsymbol{\varrho} \otimes \boldsymbol{\varrho}}{\varrho^{2}}\right] \mathrm{e}^{\mathrm{i} \varrho \omega / c}
\end{aligned}
$$

with $f(x)=x+\mathrm{i} x^{2}-x^{3}$ and $g(x)=x+3 \mathrm{i} x^{2}-3 x^{3}$.

The Hamaker approach only uses the first term of the Born series expansion. Because the Born series is a perturbative expansion in the susceptibility $\chi(\omega)$, it converges badly for materials with a large susceptibility such as gold. To improve this convergence, and to implement a local-field correction, the reference Green tensor is separated into a regular $\mathbf{R}$ and a singular part $\mathbf{D}=-\frac{c^{2}}{3 \omega^{2}} \boldsymbol{\delta}(\varrho)$. Inserting this into Eq. (A8) and performing the integrals over the $\delta$ distributions yields the local-field-corrected firstorder scattering Green tensor

$$
\begin{aligned}
\mathbf{G}^{(1)}(\boldsymbol{r}, \boldsymbol{r}, \omega)= & \frac{\omega^{2}}{c^{2}} \chi(\omega) \sum_{n=0}^{\infty}\left(-\frac{1}{3} \chi(\omega)\right)^{n} \\
& \times \int \mathrm{d}^{3} s \mathbf{R}^{(0)}(\boldsymbol{r}, \boldsymbol{s}, \omega) \mathbf{R}^{(0)}(\boldsymbol{s}, \boldsymbol{r}, \omega),
\end{aligned}
$$

which leads to

$$
\begin{aligned}
\mathbf{G}^{(1)}(\boldsymbol{r}, \boldsymbol{r}, \omega)= & \frac{\omega^{2}}{c^{2}} \frac{\chi(\omega)}{1+\chi(\omega) / 3} \\
& \times \int \mathrm{d}^{3} s \mathbf{R}^{(0)}(\boldsymbol{r}, \boldsymbol{s}, \omega) \mathbf{R}^{(0)}(\boldsymbol{s}, \boldsymbol{r}, \omega)
\end{aligned}
$$

with $(\varrho=r-s, \varrho=|\varrho|)$

$$
\mathbf{R}^{(0)}(\boldsymbol{\varrho}, \omega)=\frac{\omega}{4 \pi c}\left[f\left(\frac{c}{\omega \varrho}\right) \mathbf{I}-g\left(\frac{c}{\omega \varrho}\right) \frac{\boldsymbol{\varrho} \otimes \varrho}{\varrho^{2}}\right] \mathrm{e}^{\mathrm{i} \varrho \omega / c} .
$$

Equation (A11) has to be evaluated numerically. Because of the smooth shape of the potential, numerical integration methods converge quickly, with errors proportional to, at most, the curvature of the potential, which is small in the range of investigation.

In the Hamaker approach (pairwise summation), the potential is clearly additive. The total potential can be separated into three parts: one related to the evanescent field, one related to the dispersion interaction between $\mathrm{Rb}$ and $\mathrm{Au}$, and one part for the Casimir-Polder interaction between the $\mathrm{Rb}$ atoms and the sapphire substrate. The latter can be neglected because the interaction with the gold stripes by far dominates every other $\mathrm{CP}$ interaction, as we have explicitly checked.

\section{BEC preparation}

The BEC is prepared in an ultrahigh vacuum chamber. After precooling and trapping ${ }^{87} \mathrm{Rb}$ atoms in a magnetooptic trap, the atoms are adiabatically transferred into a highly compressed magnetic trap, where they are further cooled by forced radio-frequency evaporation by which Bose-Einstein condensates with, typically, $2 \times 10^{5}$ atoms are generated. This is done at a distance of several hundred $\mu \mathrm{m}$ from the surface of a glass prism. A thin sapphire substrate containing the gold grating investigated in this paper is glued to the top of this prism. After preparation of the BEC, we suddenly displace the magnetic trapping minimum by a distance $\Delta z$, thereby raising the potential energy of the atoms by $\Delta E=\frac{1}{2} m \omega^{2} \Delta z^{2}$, with atomic mass $m$ and magnetic trapping frequency $\omega$. The BEC is accelerated towards the new trapping minimum. After a quarter of the oscillation period $\delta t=\frac{\pi}{2 \omega}$, we switch off the magnetic trap and apply a constant magnetic-field gradient of $B^{\prime}=15 \mathrm{Gcm}^{-1}$, which compensates the gravitational force. Thus, the atoms are not further accelerated or 
decelerated because of gravitation while moving towards the grating. We measure the actual velocity of the atoms by taking absorption images of the cloud at several time intervals of $1 \mathrm{~ms}$ after the acceleration.

\section{Comparison between amplitude and phase modulation}

Experimental data are evaluated in this paper, for simplicity, only with an amplitude modulation model. This is, as will be shown below, a good approximation for the small laser powers used, whereas for large laser powers, the phase of the matter wave is also significantly modulated, leading to an increased diffraction as compared to amplitude modulation alone. For a normal incidence of the matter wave on the grating (like in the experiment-see also Fig. 2), the diffracted intensity is given within the Fraunhofer limit as [57]

$$
I(\kappa)=I_{0} k^{2}\left|\int_{-\infty}^{\infty} e^{-i \kappa x} r(x) d x\right|^{2},
$$

where $k=2 \pi / \lambda_{d B}$ with de Broglie wavelength $\lambda_{d B}=\frac{h}{m v}$ of the matter wave and $\kappa=k \cdot \sin \left(\theta_{r}\right)$ with angle $\theta_{r}$ of the reflected wave. Diffraction order $n$ is reflected at the angle $\theta_{r}^{(n)} \approx n \frac{\lambda_{d B}}{d}$. For the estimation of the relevance of phase imprinting, only relative occupations are important. Thus, neglecting prefactors in Eq. (A13), we calculate the Fourier transform $\hat{r}(\kappa)$ of the complex reflection function $r(x)$ and evaluate $\hat{r}(\kappa)$ at the values corresponding to the diffraction orders. The reflection function $r(x)=$ $r_{A}(x) \cdot e^{i \phi(x)}$ contains both amplitude and phase modulation. The phase at a certain lateral position $x$ is calculated from the simulated combined EW and CP potential landscape via [58]

$$
\phi(x)=-\frac{2}{\hbar v} \int_{+\infty}^{z_{R}(x)}\left(U_{\mathrm{EW}}(x)+U_{\mathrm{CP}}(x)\right) d z
$$

where the integral is evaluated from distances far from the surface $(z \rightarrow+\infty)$ to the distance $z_{R}(x)$, where the potential energy $U_{\mathrm{EW}}+U_{\mathrm{CP}}$ equals the kinetic energy of the atoms $E_{\text {kin }}=\frac{1}{2} m v^{2}$. The factor of 2 before the integral stems from the fact that the incoming and the reflected matter wave acquire the same phase. The amplitude of the reflection function is $r_{A}(x)=1$ for those lateral positions where atoms are reflected and $r_{A}(x)=0$ for those positions where they are transmitted. Please note that Eq. (A14) is the phase along the classical path in the eikonal approximation and that it neglects the influence of the curvature of the potential on the path. The expressions above are thus not a full solution to the scattering problem, but they are a reasonable approximation in the limit of small scattering angles $\left(\theta_{r}^{(1)} \approx 8^{\circ}\right)$. Solving Eq. (A13), we deduce the influence of phase

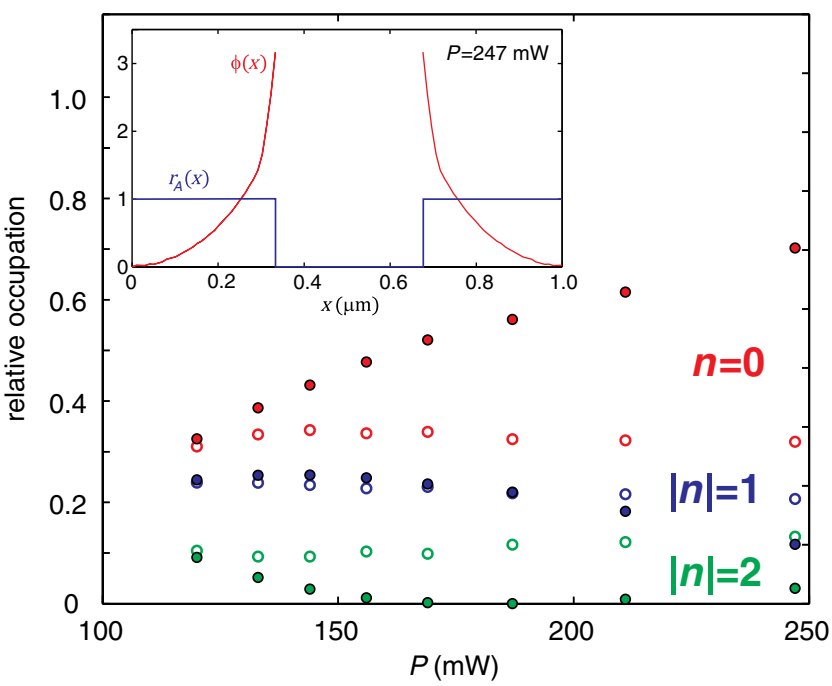

FIG. 5 Occupation of diffraction orders including phase imprinting (open circles) as compared to amplitude imprinting alone (dots). The deviation due to phase imprinting is increasing with the laser power. The inset shows the spatial dependence of the amplitude $r_{A}(x)$ and the phase $\phi(x)$ of the reflection function for a laser power of $P=247 \mathrm{~mW}$, exemplarily.

imprinting on the diffraction by comparing the results of the full reflection function $r(x)$ with those where the phase is artificially set to be constant, $\phi(x)=0$. The corresponding occupations are shown in Fig. 5. For laser powers $P<140 \mathrm{~mW}$, the deviation is $\lesssim 20 \%$ such that the diffraction can be described in some approximation by amplitude modulation alone. In contrast, this approximation is not valid for the high laser powers used where the deviation exceeds $50 \%$.

[1] J. E. Lennard Jones, Processes of Adsorption and Diffusion on Solid Surfaces, Trans. Faraday Soc. 28, 333 (1932).

[2] H. B. G. Casimir and D. Polder, The Influence of Retardation on the London-van der Waals Forces, Phys. Rev. 73, 360 (1948).

[3] S. Scheel and S. Y. Buhmann, Macroscopic Quantum Phenomena-Concepts and Applications, Acta Phys. Slovaca 58, 675 (2008).

[4] C. I. Sukenik, M. G. Boshier, D. Cho, V. Sandoghdar, and E. A. Hinds, Measurement of the Casimir-Polder Force, Phys. Rev. Lett. 70, 560 (1993).

[5] A. Landragin, J.-Y. Courtois, G. Labeyrie, N. Vansteenkiste, C. I. Westbrook, and A. Aspect, Measurement of the van der Waals Force in an Atomic Mirror, Phys. Rev. Lett. 77, 1464 (1996).

[6] A. Landragin, L. Cognet, G. Zs. K. Horvath, C. I. Westbrook, N. Westbrook, and A. Aspect, A Reflection Grating for Atoms at Normal Incidence, Europhys. Lett. 39, 485 (1997). 
[7] F. Shimizu, Specular Reflection of Very Slow Metastable Neon Atoms from a Solid Surface, Phys. Rev. Lett. 86, 987 (2001).

[8] V. Druzhinina and M. DeKieviet, Experimental Observation of Quantum Reflection Far from Threshold, Phys. Rev. Lett. 91, 193202 (2003).

[9] T. A. Pasquini, Y. Shin, C. Sanner, M. Saba, A. Schirotzek, D. E. Pritchard, and W. Ketterle, Quantum Reflection from a Solid Surface at Normal Incidence, Phys. Rev. Lett. 93, 223201 (2004).

[10] J. M. Obrecht, R. J. Wild, M. Antezza, L. P. Pitaevskii, S. Stringari, and E. A. Cornell, Measurement of the Temperature Dependence of the Casimir-Polder Force, Phys. Rev. Lett. 98, 063201 (2007).

[11] H. Bender, P. W. Courteille, C. Marzok, C. Zimmermann, and S. Slama, Direct Measurement of Intermediate-Range Casimir-Polder Potentials, Phys. Rev. Lett. 104, 083201 (2010).

[12] R. Messina, D. A. R. Dalvit, P. A. Maia Neto, A. Lambrecht, and S. Reynaud, Dispersive Interactions between Atoms and Nonplanar Surfaces, Phys. Rev. A 80, 022119 (2009).

[13] A. W. Rodriguez, F. Capasso, and S. G. Johnson, The Casimir Effect in Microstructured Geometries, Nat. Photonics 5, 211 (2011).

[14] V. N. Marachevsky, The Casimir Effect: Medium and Geometry, J. Phys. A 45, 374021 (2012).

[15] M. Levin, A. P. McCauley, A. W. Rodriguez, M. T. Homer Reid, and S. G. Johnson, Casimir Repulsion between Metallic Objects in Vacuum, Phys. Rev. Lett. 105, 090403 (2010).

[16] F. W. DelRio, M. P. de Boer, J. A. Knapp, E. D. Reedy Jr., P. J. Clews, and M. L. Dunn, The Role of van der Waals Forces in Adhesion of Micromachined Surfaces, Nat. Mater. 4, 629 (2005).

[17] A. Lambrecht and V. N. Marachevsky, Casimir Interaction of Dielectric Gratings, Phys. Rev. Lett. 101, 160403 (2008); Theory of the Casimir Effect in One-Dimensional Periodic Dielectric Systems, Int. J. Mod. Phys. A 24, 1789 (2009).

[18] A. M. Contreras-Reyes, R. Guérout, P. A. Maia Neto, D. A. R. Dalvit, A. Lambrecht, and S. Reynaud, Casimir-Polder Interaction between an Atom and a Dielectric Grating, Phys. Rev. A 82, 052517 (2010).

[19] P. S. Davids, F. Intravaia, F. S. S. Rosa, and D. A. R. Dalvit, Modal Approach to Casimir Forces in Periodic Structures, Phys. Rev. A 82, 062111 (2010).

[20] D. Farías and K.-H. Rieder, Atomic Beam Diffraction from Solid Surfaces, Rep. Prog. Phys. 61, 1575 (1998).

[21] T. A. Pasquini, M. Saba, G.-B. Jo, Y. Shin, W. Ketterle, D. E. Pritchard, T. A. Savas, and N. Mulders, Low Velocity Quantum Reflection of Bose-Einstein Condensates, Phys. Rev. Lett. 97, 093201 (2006).

[22] B. S. Zhao, S. A. Schulz, S. A. Meek, G. Meijer, and W. Schöllkopf, Quantum Reflection of Helium Atom Beams from a Microstructured Grating, Phys. Rev. A 78, 010902 (R) (2008).

[23] R. E. Grisenti, W. Schöllkopf, J. P. Toennies, G. C. Hegerfeldt, and T. Köhler, Determination of Atom-Surface van der Waals Potentials from Transmission-Grating Diffraction Intensities, Phys. Rev. Lett. 83, 1755 (1999).
[24] H. Oberst, D. Kouznetsov, K. Shimizu, J. I. Fujita, and F. Shimizu, Fresnel Diffraction Mirror for an Atomic Wave, Phys. Rev. Lett. 94, 013203 (2005).

[25] J. D. Perreault and A. D. Cronin, Observation of Atom Wave Phase Shifts Induced by van der Waals Atom-Surface Interactions, Phys. Rev. Lett. 95, 133201 (2005).

[26] G. A. Moreno, D. A. R. Dalvit, and E. Calzetta, Bragg Spectroscopy for Measuring Casimir-Polder Interactions with Bose-Einstein Condensates above Corrugated Surfaces, New J. Phys. 12, 033009 (2010).

[27] C. Stehle, H. Bender, C. Zimmermann, D. Kern, M. Fleischer, and S. Slama, Plasmonically Tailored Micropotentials for Ultracold Atoms, Nat. Photonics 5, 494 (2011).

[28] M. Righini, A. S. Zelenina, C. Girard, and R. Quidant, Parallel and Selective Trapping in a Patterned Plasmonic Landscape, Nat. Phys. 3, 477 (2007).

[29] S. Y. Buhmann, L. Knöll, D. G. Welsch, and H. T. Dung, Casimir-Polder Forces: A Nonperturbative Approach, Phys. Rev. A 70, 052117 (2004).

[30] J. H. de Boer, The Influence of van der Waals' Forces and Primary Bonds on Binding Energy, Strength and Orientation, with Special Reference to Some Artificial Resins, Trans. Faraday Soc. 32, 10 (1936).

[31] H. C. Hamaker, The London van der Waals Attraction between Spherical Particles, Physica (Amsterdam) 4, 1058 (1937).

[32] S. N. Thennadil and L. H. Garcia-Rubio, Approximations for Calculating van der Waals Interaction Energy between Spherical Particles - A Comparison, J. Colloid Interface Sci. 243, 136 (2001).

[33] H.-C. Chiu, G. L. Klimchitskaya, V. N. Marachevsky, V. M. Mostepanenko, and U. Mohideen, Demonstration of the Asymmetric Lateral Casimir Force between Corrugated Surfaces in the Nonadditive Regime, Phys. Rev. B 80, 121402(R) (2009); Lateral Casimir Force between Sinusoidally Corrugated Surfaces: Asymmetric Profiles, Deviations from the Proximity Force Approximation, and Comparison with Exact Theory, 81, 115417 (2010).

[34] S. Y. Buhmann and D.-G. Welsch, Born Expansion of the Casimir-Polder Interaction of a Ground-State Atom with Dielectric Bodies, Appl. Phys. B 82, 189 (2006).

[35] V. A. Parsegian, Van der Waals Forces: A Handbook for Biologists, Chemists, Engineers, and Physicists (Cambridge University Press, New York, 2005).

[36] K. Autumn, Y. A. Liang, S. T. Hsieh, W. Zesch, W. P. Chan, T. W. Kenny, R. Fearing, and R. J. Full, Adhesive Force of a Single Gecko Foot-Hair, Nature (London) 405, 681 (2000).

[37] R. B. Rodrigues, P. A. Maia Neto, A. Lambrecht, and S. Reynaud, Lateral Casimir Force beyond the ProximityForce Approximation, Phys. Rev. Lett. 96, 100402 (2006).

[38] A. Rodriguez, M. Ibanescu, D. Iannuzzi, F. Capasso, J. D. Joannopoulos, and S. G. Johnson, Computation and Visualization of Casimir Forces in Arbitrary Geometries: Nonmonotonic Lateral-Wall Forces and the Failure of Proximity-Force Approximations, Phys. Rev. Lett. 99, 080401 (2007). 
[39] Y. Bao, R. Guérout, J. Lussange, A. Lambrecht, R. A. Cirelli, F. Klemens, W. M. Mansfield, C. S. Pai, and H. B. Chan, Casimir Force on a Surface with Shallow Nanoscale Corrugations: Geometry and Finite Conductivity Effects, Phys. Rev. Lett. 105, 250402 (2010).

[40] L. P. Teo, M. Bordag, and V. Nikolaev, Corrections beyond the Proximity Force Approximation, Phys. Rev. D 84, 125037 (2011).

[41] G. Bimonte, T. Emig, R. L. Jaffe, and M. Kardar, Casimir Forces beyond the Proximity Approximation, Europhys. Lett. 97, 50001 (2012).

[42] A. D. Cronin and J. D. Perreault, Phasor Analysis of Atom Diffraction from a Rotated Material Grating, Phys. Rev. A 70, 043607 (2004).

[43] C. Henkel, J.-Y. Courtois, and A. Aspect, Atomic Diffraction by a Thin Phase Grating, J. Phys. II France 4, 1955 (1994).

[44] A. Günther, S. Kraft, C. Zimmermann, and J. Fortágh, Atom Interferometer Based on Phase Coherent Splitting of Bose-Einstein Condensates with an Integrated Magnetic Grating, Phys. Rev. Lett. 98, 140403 (2007).

[45] A. D. Cronin, J. Schmiedmayer, and D. E. Pritchard, Optics and Interferometry with Atoms and Molecules, Rev. Mod. Phys. 81, 1051 (2009).

[46] J. M. McGuirk, D. M. Harber, J. M. Obrecht, and E. A. Cornell, Alkali-Metal Adsorbate Polarization on Conducting and Insulating Surfaces Probed with Bose-Einstein Condensates, Phys. Rev. A 69, 062905 (2004).

[47] C. Stehle, H. Bender, F. Jessen, C. Zimmermann, and S. Slama, Ad-and Desorption of Rb Atoms on a Gold Nanofilm Measured by Surface Plasmon Polaritons, New J. Phys. 12, 083066 (2010).

[48] A. Tauschinsky, R. M. T. Thijssen, S. Whitlock, H. B. van Linden van den Heuvell, and R. J. C. Spreeuw, Spatially Resolved Excitation of Rydberg Atoms and Surface Effects on an Atom Chip, Phys. Rev. A 81, 063411 (2010).
[49] H. Failache, S. Saltiel, M. Fichet, D. Bloch, and M. Ducloy, Resonant van der Waals Repulsion between Excited Cs Atoms and Sapphire Surface, Phys. Rev. Lett. 83, 5467 (1999).

[50] F. Intravaia, C. Henkel, and A. Lambrecht, Role of Surface Plasmons in the Casimir Effect, Phys. Rev. A 76, 033820 (2007).

[51] K. A. Milton, E. K. Abalo, P. Parashar, N. Pourtolami, I. Brevik, and S. Å. Ellingsen, Casimir-Polder Repulsion near Edges: Wedge Apex and a Screen with an Aperture, Phys. Rev. A 83, 062507 (2011).

[52] K. A. Milton, P. Parashar, N. Pourtolami, and I. Brevik, Casimir-Polder Repulsion: Polarizable Atoms, Cylinders, Spheres, and Ellipsoids, Phys. Rev. D 85, 025008 (2012).

[53] C. Eberlein and R. Zietal, Casimir-Polder Interaction between a Polarizable Particle and a Plate with a Hole, Phys. Rev. A 83, 052514 (2011).

[54] M. S. Safronova, C. J. Williams, and C. W. Clark, Relativistic Many-Body Calculations of Electric-Dipole Matrix Elements, Lifetimes, and Polarizabilities in Rubidium, Phys. Rev. A 69, 022509 (2004).

[55] D. A. Steck, Rubidium 87D Line Data, http://steck.us/ alkalidata (2009).

[56] A. A. Maradudin and D. L. Mills, Scattering and Absorption of Electromagnetic Radiation by a Semi-infinite Medium in the Presence of Surface Roughness, Phys. Rev. B 11, 1392 (1975); D. L. Mills and A. A. Maradudin, Surface Roughness and the Optical Properties of a Semi-infinite Material: The Effect of a Dielectric Overlayer, Phys. Rev. B 12, 2943 (1975).

[57] R. E. Grisenti, W. Schöllkopf, J. P. Toennies, J. R. Manson, T. A. Savas, and H. I. Smith, He-atom Diffraction from Nanostructure Transmission Gratings: The Role of Imperfections, Phys. Rev. A 61, 033608 (2000).

[58] V. P. A. Lonij, W. F. Holmgren, and A. D. Cronin, Magic Ratio of Window Width to Grating Period for van der Waals Potential Measurements Using Material Gratings, Phys. Rev. A 80, 062904 (2009). 